\title{
Conhecimentos e práticas de profissionais sobre desenvolvimento da criança na Atenção Básica à Saúde
}

\author{
Knowledge and practices regarding child development among primary healthcare professionals
}

\author{
Augusta Morgado Ribeiro¹, Rosa Resegue F. da Silva², Rosana Fiorini Puccini ${ }^{3}$
}

\section{RESUMO}

Objetivo: Avaliar o conhecimento e as práticas sobre desenvolvimento infantil de médicos que atuam em Unidades Básicas de Saúde (UBS).

Método: Estudo transversal, descritivo, realizado nas UBS de Embu (SP). Procedimentos do estudo: 1) avaliação do conhecimento por teste contendo 20 questões de múltipla escolha sobre desenvolvimento da criança aplicado a 31 médicos (universo) que prestam assistência pediátrica em UBS; 2) avaliação das práticas - entrevista semiestruturada aplicada para uma amostra de 154 mães/cuidadores que acompanhavam crianças com idade menor ou igual a 36 meses em consulta médica agendada em UBS do município. Para comparação de variáveis categóricas (avaliação/orientações sobre desenvolvimento em consultas de crianças de diferentes faixas etárias), utizou-se o qui-quadrado.

Resultados: A média de acertos dos médicos foi de 14,8 questões; sete questões apresentaram índices de erros superiores a $30 \%$ (desenvolvimento sensorial, aquisição de linguagem, fisiologia do sistema nervoso, diagnóstico clínico e laboratorial de infecções congênitas, erros inatos do metabolismo) e quatro questões apresentaram acertos acima de $85 \%$ (marcos do desenvolvimento motor, pessoal-social, fatores de risco e síndrome genética). Quanto às práticas, em 69 (45\%) consultas o médico perguntou a opinião da mãe/ cuidador sobre o desenvolvimento da criança, em 80 (52\%) a mãe/cuidador referiu que o médico fez alguma pergunta e/ ou avaliou o desenvolvimento e em 64 (42\%) orientou sobre como estimular a criança.
Conclusões: Identificaram-se falhas de conhecimento e nas práticas dos profissionais referentes ao desenvolvimento da criança, o que indica a necessidade de implementar educação permanente.

Palavras-chave: desenvolvimento infantil; avaliação de Serviços de Saúde; cuidados primários de saúde.

\section{ABSTRACT}

Objective: To evaluate the knowledge and practices regarding child development among physicians working in primary healthcare units.

Method: Cross-sectional descriptive study carried out at primary healthcare units in Embu, São Paulo, Brazil. Study procedures: 1) Evaluation of knowledge: test consisting of 20 multiple-choice questions on child development applied to all 31 physicians who were providing pediatric care at the primary healthcare units; 2) Evaluation of practices: semistructured interview applied to a sample of 154 mothers/ caregivers of children aged up to 36 months during followup visits at primary healthcare units in the municipality. For the comparisons of categorical variables (evaluation/advices about development in visits of children at different ages), the chi-square test was employed.

Results: The mean number of correct responses among physicians was 14.8. The error rate for seven questions was greater than $30 \%$ (sensory development, language acquisition, physiology of the nervous system, clinical and laboratory diagnosis of congenital infections and innate errors of
Instituição: Universidade Federal de São Paulo (Unifesp), São Paulo, SP, Brasil

${ }^{1}$ Estudante de Graduação do Curso de Medicina da Unifesp, São Paulo, SP, Brasil

${ }^{2}$ Doutora; Coordenadora do Projeto Desenvolver da Unifesp, Secretaria Municipal de Saúde do Embu; Pediatra da Disciplina de Pediatria Geral e Comunitária do Departamento de Pediatria da Unifesp, São Paulo, SP, Brasil

${ }^{3}$ Livre-docente; Professora Titular da Disciplina de Pediatria Geral e Comunitária do Departamento de Pediatria da Unifesp, São Paulo, SP, Brasil
Endereço para correspondência:

Rosana Fiorini Puccini

Rua Botucatu, 598 - Vila Clementino

CEP 04023-062 - São Paulo/SP

E-mail: rpuccini@terra.com.br

Conflitos de interesse: nada a declarar.

Fonte financiadora: o primeiro autor foi bolsista de Iniciação Científica do CNPq 2007-8

Recebido em: 22/5/09

Aprovado em: 8/9/09 
metabolism) and the rate of correct responses was greater than $85 \%$ for four questions (motor and personal-social development markers, risk factors and genetic syndromes). Regarding practices, in 69 (45\%) visits, the doctor asked the mother/caregiver's opinion about the child's development; in $80(52 \%)$, the mother/caregiver said that the doctor assessed the development; and in 64 (42\%), the mother/ caregiver said that the doctor advised them on practices for child's stimulation.

Conclusions: Faulty knowledge and practices regarding child development were identified among primary care professionals, indicating the need for continued education.

Key-words: child development; Health Services evaluation; primary health care.

\section{Introdução}

A vigilância do desenvolvimento é um eixo integrador da atenção à saúde da criança, compreendendo as atividades relacionadas à promoção do desenvolvimento normal e à detecção de desvios nesse processo. Estima-se que uma em cada oito crianças apresenta alterações do desenvolvimento que interferem de forma significativa em sua qualidade de vida e inclusão na sociedade ${ }^{(1)}$. O diagnóstico e a intervenção precoces, antes dos cinco anos de idade, são decisivos para o prognóstico de desenvolvimento dessas crianças ${ }^{(2)}$.

Os primeiros anos de vida são particularmente importantes porque é quando ocorrem processos vitais do desenvolvimento, em todos os domínios de funções. Durante esse período, graças a uma intensa atividade cerebral, fruto da interação entre as características biológicas e as oportunidades de experiência dos indivíduos, desenvolvem-se funções específicas, cada uma delas embasando o processo de desenvolvimento de outros domínios e a aquisição de atividades mais complexas ${ }^{(3)}$. A despeito da maior vulnerabilidade desse período, a intensa neuroplasticidade é também responsável por melhores possibilidades prognósticas, se a intervenção ocorrer precocemente ${ }^{(1,4)}$.

$\mathrm{Na}$ maioria das situações, o pediatra é o primeiro e único profissional a entrar em contato com essas crianças, portanto, é essencial que esteja capacitado a detectar as alterações apresentadas, garantindo intervenções oportunas ${ }^{(5-7)}$. De acordo com Halfon et al, o acompanhamento do desenvolvimento permite a identificação e o tratamento desses distúrbios, prevenindo-se a perda de seu potencial ${ }^{(8)}$. Idealmente, avaliações realizadas no momento apropriado podem ajudar a identificar fatores de risco para o desenvolvimento, tornando possível uma pré-orientação que forneça aos pais estratégias para a sua promoção. A avaliação do desenvolvimento pelo profissional de forma compartilhada com seus familiares representa, ainda, um processo educativo, promovendo uma oportunidade para os pediatras auxiliarem os pais a compreenderem aspectos relacionados ao desenvolvimento, ressaltando características do processo normal e reformulando percepções desajustadas e inapropriadas sobre comportamento.

O acompanhamento de crianças e do processo de desenvolvimento reúne diferentes modalidades de avaliação, as quais incluem a percepção dos pais, professores, pediatras e demais profissionais de saúde. Nesse processo, têm sido consideradas a anamnese, a observação da criança em seu ambiente, na prática de atividades ou, ainda, a utilização de instrumentos de rastreamento ${ }^{(9,10)}$. A triagem neonatal para erros inatos do metabolismo, a inclusão rotineira da triagem auditiva e da identificação do reflexo vermelho nas maternidades são instrumentos importantes para detectar condições que cursam com alterações do desenvolvimento e devem ser associados ao cuidadoso acompanhamento da criança, pois, além de possíveis falhas nesses exames, existe a possibilidade de doenças adquiridas e de alterações em outras funções do desenvolvimento ${ }^{(11,12)}$.

São poucos os estudos que abordam o conhecimento e as práticas dos profissionais em relação ao desenvolvimento da criança em nosso país ${ }^{(13,14)}$. A Universidade Federal de São Paulo (Unifesp) atua no município do Embu (SP) desde 1970, com expressiva participação na assistência, planejamento das ações em saúde e programas de educação permanente ${ }^{(15)}$. Em 1995, foi criado um ambulatório multiprofissional - Projeto Desenvolver - direcionado à criança com risco de apresentar atraso do desenvolvimento ou com atraso estabelecido ${ }^{(16)}$. Esse ambulatório conta com profissionais da universidade e da secretaria municipal de saúde (saúde mental, pediatria, fonoaudiologia, tecnologia oftálmica, fisioterapia, neuropediatria), constituindo referência para o município. Como desdobramentos desse trabalho, têm sido realizados cursos e capacitações sobre desenvolvimento infantil para os profissionais que prestam assistência pediátrica no município. Entretanto, verifica-se que crianças com atrasos de desenvolvimento ainda são encaminhadas tardiamente para este ambulatório. Assim, o objetivo deste estudo foi avaliar o conhecimento dos profissionais sobre desenvolvimento da criança assim como suas práticas, ou seja, se estes profissionais buscam obter informações sobre o desenvolvimento da criança junto à mãe/cuidador que acompanha a criança e se 
são realizadas avaliação e orientações nas consultas agendadas. Tais resultados deverão constituir importantes subsídios para ações direcionadas ao aprimoramento do Programa Integral de Atenção à Criança no município, considerando a importância da detecção precoce desses distúrbios e intervenções oportunas.

\section{Métodos}

Trata-se de estudo transversal e descritivo, realizado no município do Embu. Embu está situado na região metropolitana de São Paulo e, em 2007, sua população foi estimada em 249.777 habitantes. O Índice de Desenvolvimento Humano Municipal (IDHM) foi 0,772, inferior à média do Estado de São Paulo $(0,814)$ nesse mesmo ano; a taxa de mortalidade infantil do município foi de 12,0 por mil nascidos vivos, também em 2007, sendo que a principal causa de óbito foram as afecções perinatais, seguidas de malformações congênitas e doenças do aparelho respiratório ${ }^{(17)}$. A rede de saúde municipal é constituída por 13 unidades básicas de saúde (UBS), dois prontos-socorros, uma maternidade (gestações/parto de médio e baixo risco), ambulatório de especialidades e de saúde mental e hospital regional de 250 leitos, responsável pela assistência à gestações/partos de alto risco.

No município, o Programa de Atenção Integral à Saúde da Criança, desenvolvido segundo critérios de risco para morbidade e mortalidade, conta com dois cronogramas ${ }^{(18)}$ de atendimentos para crianças de baixo risco (BR) e alto risco (AR), conforme descritos na Figura 1. As atividades incluem consultas médicas e atendimentos de enfermagem e, desde 2007, foi integrada ao programa a consulta do binômio mãecriança, realizada nos primeiros 15 dias de vida da criança por profissionais de enfermagem, sendo que recém-nascido e puérpera recebem alta da maternidade com a consulta já agendada. As demais consultas de enfermagem são realizadas em grupos de seis a oito mães/crianças, organizados por faixa etária; nestas consultas as crianças têm seu peso e estatura aferidos, sendo avaliada a alimentação, a imunização e o

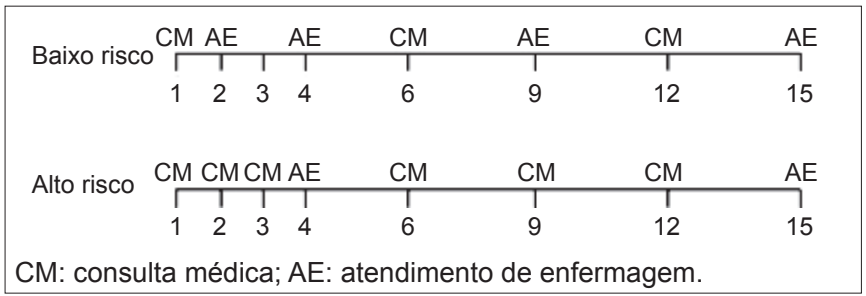

Figura 1 - Cronograma de atendimento, vigilância do crescimento e desenvolvimento, Secretaria de Saúde do Embu. desenvolvimento, por meio de roteiro definido com marcos do desenvolvimento para cada faixa etária. São realizadas orientações apropriadas para cada faixa etária e, sempre que necessário, é feito o encaminhamento para o médico, independentemente do cronograma. Após os 15 meses, as mães são orientadas a agendarem consultas de rotina a cada seis meses ou sempre que considerarem necessário. Crianças com afecções crônicas ou outras condições que requeiram consultas/avaliações específicas de médicos ou de outros profissionais têm seu agendamento estabelecido de acordo com suas necessidades individuais. Essas ações são complementadas com outras atividades desenvolvidas na unidade: acolhimento e atendimento médico para consultas não agendadas, programa de atenção à criança com asma brônquica, ambulatório de dificuldades escolares e programa de atenção à criança com risco para atraso de desenvolvimento e com atraso já estabelecido.

Assim o presente estudo foi feito nas Unidades Básicas de Saúde do município do Embu, sendo incluídos tanto o universo de médicos que prestam assistência à criança na Rede Básica de Saúde do Embu (31 profissionais), como uma amostra por conveniência de acompanhantes (mães ou cuidadores) de crianças com idade menor ou igual a 36 meses que haviam comparecido a consultas médicas agendadas de seis unidades básicas de saúde (25 mães/cuidadores por Unidade Básica de Saúde). Estas seis unidades são responsáveis pelo atendimento de aproximadamente $75 \%$ da população do município; as outras sete são unidades menores, organizadas segundo o modelo assistencial do Programa de Saúde da Família, com agendamento não-específico para Pediatria em cada período. O número de entrevistas em cada uma das seis unidades variou de 23 a 29 e a amostra final contou com 154 entrevistas, realizadas no período de março a dezembro de 2008, em diferentes dias/horários da semana, visando a incluir o maior número de profissionais da respectiva unidade. A amostra de 154 entrevistas foi referente às consultas realizadas por 12 profissionais, dentre 14 existentes nessas unidades, e pela equipe da Unifesp (internos da $5^{\text {a }}$ série do curso de medicina e residentes do $1^{\circ}$ ano, sob supervisão docente).

Para a coleta de dados, aplicou-se um teste de múltipla escolha para os médicos e uma entrevista semiestruturada para as mães/acompanhantes. O teste de múltipla escolha era composto por 20 questões, com quatro alternativas que englobavam conteúdos relacionados ao processo e fisiologia do desenvolvimento, avaliação e marcos do desenvolvimento, segundo os domínios de função pessoal- 
social, motor grosseiro, motor adaptativo e de linguagem, diagnóstico clínico e laboratorial de erros inatos do metabolismo e de infecções congênitas. Este teste, elaborado pela equipe multiprofissional da Unifesp envolvida no Projeto Desenvolver, já havia sido utilizado em dois estudos realizados em Belém do Paráa ${ }^{(13,19)}$. No município do Embu, o teste foi aplicado no $2^{\circ}$ semestre de 2007 para todos os médicos que realizam assistência às crianças nas UBSs, por ocasião de realização do programa de educação permanente em Pediatria. Para aqueles que faltaram a essa atividade, o teste foi aplicado individualmente. Foram obtidas, ainda, informações referentes à idade, ano de formatura, residência médica ou especialização e tipos de serviço nos quais atua além da UBS (unidades de emergência, de internação ou ambulatórios). A identificação do profissional foi facultativa.

A entrevista semiestruturada foi aplicada aos acompanhantes de crianças de 0 a 36 meses em consulta pediátrica agendada, após o seu término. Nela, perguntou-se a opinião das mães ou cuidadores sobre o desenvolvimento da sua criança, bem como os procedimentos do médico em relação a esse desenvolvimento. As entrevistas incluíam perguntas objetivas ( $\operatorname{sim} /$ não) e discursivas sobre o que foi observado pelos entrevistados durante a consulta médica. As variáveis questionadas incluíam: idade da criança, opinião da mãe/cuidador sobre o desenvolvimento da criança (normal, atrasado ou adiantado), se o profissional perguntou sobre sua opinião em relação ao desenvolvimento da sua criança ( $\operatorname{sim} /$ não), se o profissional fez perguntas para avaliar o desenvolvimento (sim/não e se respondeu sim, quais foram as perguntas, sugerindose alguns marcos do desenvolvimento para exemplificar), se o profissional orientou sobre como estimular a criança ( $\operatorname{sim} /$ não e se respondeu sim, quais foram as orientações). Essas entrevistas foram realizadas pela aluna bolsista em duas unidades de saúde e por profissional auxiliar de enfermagem previamente capacitado que atua no Projeto Desenvolver, nas outras quatro unidades.

Para o processamento do texto, entrada e análise descritiva dos dados foi utilizado o Programa Excel. Na comparação de variáveis categóricas, aplicou-se o teste qui-quadrado (EpiInfo 6.01). O desfecho esperado era: resposta positiva (sim) para as Perguntas 2 (perguntou a opinião da mãe sobre o desenvolvimento), 3 (perguntou/avaliou o desenvolvimento na consulta) e 4 (orientou como estimular a criança), segundo as seguintes faixas etárias: idade menor ou igual a 12 meses e maior que 12 meses.
A realização do projeto foi autorizada pela Secretaria Municipal de Saúde do Embu, tendo sido aprovada pelo Comitê de Ética em Pesquisa da Universidade Federal de São Paulo.

\section{Resultados}

O teste de desenvolvimento com questões de múltipla escolha foi respondido por $100 \%$ dos profissionais (31 profissionais). Destes, 29 informaram o ano de sua formatura e idade; verificou-se que o número de anos de formação variou de seis a 45 anos (média de 21,3 anos) e que a idade variou de 30 a 68 anos (média de 46,7 anos). Dentre os 31 profissionais que responderam ao questionário, 24 haviam realizado residência ou especialização na área de pediatria, três em Clínica Médica e quatro em Medicina Social/Saúde Pública. Em relação a outras atividades, 17 relataram que mantêm atuação em um ou mais serviços além das UBS do município - unidades de emergência (sete), unidades de internação (três) e ambulatórios (dez).

Em relação ao desempenho no teste de múltipla escolha, obteve-se uma média de acertos de 14,8 questões (74,0\%); a distribuição de erros e acertos para cada questão encontrase na Tabela 1. Sete questões apresentaram índices de erros superiores a $30 \%$ e quatro questões apresentaram acertos acima de $85 \%$. Nenhuma questão mostrou porcentagem de erros maior que $50 \%$. Verificou-se que 18 profissionais apresentaram um desempenho superior ou igual a $70 \%$ de acertos e os demais acertaram entre $50 \%$ e $70 \%$ das questões. As Perguntas com porcentagem de erros acima de 30\% foram: 1 (caso clínico, sinais precoces de alterações do desenvolvimento visual, pessoal-social), 11 (definição dos conceitos de habilidades motoras grosseiras e finas), 13 (desenvolvimento motor - processo e fisiologia), 14 (avaliação do desenvolvimento sensorial), 15 (infecções congênitas - diagnóstico clínico e laboratorial), 16 (infecções congênitas - diagnóstico clínico) e 19 (caso clínico/ erros inatos do metabolismo). As perguntas com porcentagem de acertos superior a $85 \%$ foram: 2 (marcos do desenvolvimento - desenvolvimento motor), 6 (marcos do desenvolvimento - desenvolvimento motor/pessoal-social), 7 (fatores de risco para atraso do desenvolvimento) e 18 (caso clínico/ síndrome genética).

Todas as crianças eram matriculadas nas unidades de saúde onde estavam sendo realizadas as consultas. A primeira pergunta à mãe/cuidador referia-se à sua opinião sobre o desenvolvimento da criança: $55,7 \%$ as consideravam normais, 
$42,1 \%$ as consideravam adiantadas e apenas $2,3 \%$ consideravam que havia algum problema de desenvolvimento. Os comentários referidos pelas mães/cuidadores para justificar a sua opinião sobre esse desenvolvimento foram: sorri, grita, observa, reage ao som, fica em pé com apoio; fala, sabe nomear objetos, pula, atende telefone; fala frases, anda, corre, tira a roupa, chuta bola; vai ao banheiro sozinha, dança, come sozinha, sobe escada sozinha.

Tabela 1 - Distribuição de erros e acertos dos profissionais de saúde, por questão, em teste de múltipla escolha sobre desenvolvimento infantil, Embu, 2007-2008

\begin{tabular}{lcc}
\hline Questões & Erros & Acertos \\
\hline 1 & 10 & 21 \\
2 & 4 & 27 \\
3 & 7 & 24 \\
4 & 9 & 22 \\
5 & 7 & 24 \\
6 & 4 & 27 \\
7 & 1 & 30 \\
8 & 5 & 26 \\
9 & 6 & 25 \\
10 & 6 & 25 \\
11 & 21 & 10 \\
12 & 7 & 24 \\
13 & 13 & 18 \\
14 & 13 & 18 \\
15 & 13 & 18 \\
16 & 13 & 18 \\
17 & 8 & 23 \\
18 & 2 & 29 \\
19 & 15 & 16 \\
20 & 7 & 24 \\
\hline
\end{tabular}

A segunda e terceira perguntas referiam-se à avaliação do médico em relação ao desenvolvimento. Em 69 (44,8\%) consultas, o médico perguntou a opinião da mãe/cuidador sobre o desenvolvimento da criança e, em 80 (51,9\%) consultas, a mãe/cuidador referiu que o médico fez alguma pergunta sobre o desenvolvimento da criança. As perguntas mais frequentemente utilizadas pelos profissionais foram: se acompanha o rosto com o olhar, se fixa o olhar, se ouve bem; se anda, fala, levanta-se sozinho, sorri para as pessoas, fala frases, o que sabe fazer no dia-a-dia, se brinca, se pergunta coisas, dá tchau, bate palmas, se estranha as pessoas.

A quarta pergunta referia-se às orientações do profissional; em $64(41,6 \%)$ consultas, a mãe/cuidador relatou que o médico fez orientações sobre como estimular a criança. As orientações mais frequentes foram: brincar com a criança, dar brinquedos coloridos, bolas; estimular brincadeiras, integrar no dia-a-dia da família; cantar, conversar, tocar, utilizar sons, observar o olhar; sair com a criança, ensinar o nome correto dos objetos.

Não houve diferença estatisticamente significativa na porcentagem de consultas em que foi perguntada a opinião da mãe/cuidador, realizada a avaliação e a orientação pelo profissional segundo a faixa etária da criança (Tabela 2).

\section{Discussão}

Este estudo permitiu caracterizar o perfil do médico que presta assistência pediátrica nas UBS do município do Embu. A maioria realizou residência ou especialização em Pediatria e a média de anos de formado foi de 21,3 anos, ou seja, eram médicos experientes para executar a avaliação do desenvolvimento da criança.

Tabela 2 - Práticas dos profissionais referentes ao desenvolvimento da criança, Embu, 2008

\begin{tabular}{|c|c|c|c|c|c|c|c|}
\hline \multirow{3}{*}{ Perguntas } & \multicolumn{7}{|c|}{ Idade (meses) } \\
\hline & \multicolumn{2}{|c|}{$\leq 12 M(n=84)$} & \multicolumn{2}{|c|}{$>12 M(n=70)$} & \multirow{2}{*}{ Valor de $p$} & \multicolumn{2}{|c|}{ Total $(n=154)$} \\
\hline & $n$ & $\%$ & $n$ & $\%$ & & $n$ & $\%$ \\
\hline \multicolumn{8}{|l|}{ Pergunta 2} \\
\hline Sim & 35 & 41,7 & 34 & 48,6 & 0,39 & 69 & 44,8 \\
\hline \multicolumn{8}{|l|}{ Pergunta 3} \\
\hline Sim & 45 & 53,6 & 35 & 50,0 & 0,65 & 80 & 51,9 \\
\hline \multicolumn{8}{|l|}{ Pergunta 4} \\
\hline Sim & 35 & 41,7 & 29 & 41,4 & 0,97 & 64 & 41,6 \\
\hline
\end{tabular}

Pergunta 2 - O médico que atendeu sua criança hoje perguntou o que a senhora acha sobre o comportamento/desenvolvimento? ( ) Sim ( ) Não Pergunta 3 - O médico observou e/ou perguntou, hoje, algo sobre o comportamento da sua criança? (citados, como exemplos, os marcos que deveriam ser investigados para a faixa etária) ( ) Sim ( ) Não

Pergunta 4 - O médico orientou sobre como estimular/brincar com seu filho? ( ) Sim ( ) Não 
Quanto ao conhecimento destes profissionais, avaliado por meio do teste de questões de múltipla escolha já descrito, foi possível identificar temas que apresentaram maiores falhas de conhecimento e, portanto, que merecem reforço em programas de educação permanente. As questões com maiores índices de erros foram as que envolviam conceitos abordando a fisiopatologia de algumas alterações do desenvolvimento. As questões com maiores índices de acerto estavam mais focadas nos marcos do desenvolvimento e, portanto, englobavam os conhecimentos relacionados ao cotidiano dos profissionais na assistência pediátrica. Os resultados obtidos, tanto no que se refere ao número de acertos, como aos conteúdos de questões com maiores índices de erros, foram semelhantes à $1^{a}$ etapa do estudo realizado em Belém, em que a média de acertos foi de 13,3/19 questões ${ }^{(13)}$. Outros trabalhos que buscaram avaliar o conhecimento de médicos em relação ao desenvolvimento constataram insuficiências e necessidade de capacitação de forma continuada ${ }^{(20,21)}$.

Em relação às práticas, a análise dos resultados requer uma compreensão das limitações deste estudo. As entrevistas com as mães/cuidadores foram realizadas em diferentes dias da semana e períodos, buscando abarcar consultas realizadas pelo maior número possível de profissionais, porém, por razões operacionais, não foi estabelecida uma amostra que representasse de forma equitativa todos os profissionais do município, os quais participaram da avaliação de conhecimento.

Observou-se que cerca de metade das mães/cuidadores entrevistados referiu que o médico fez alguma pergunta ou avaliou formalmente o desenvolvimento das crianças consultadas, o que tem sido observado por outros estudos ${ }^{(8)}$. Comparando-se estes resultados aos obtidos na primeira etapa do estudo de Belém, verifica-se que as porcentagens do Embu foram superiores ${ }^{(13)}$. Em novo estudo realizado em Belém, os mesmos autores constataram que os médicos mantiveram os mesmos índices de acertos obtidos logo após o programa de educação continuada e que houve melhora das práticas durante a consulta, resultados atribuídos ao programa de educação permanente e, principalmente, ao fato de ter sido estruturado um fluxo adequado dos casos com suspeita e/ou atraso do desenvolvimento detectados pelos profissionais da atenção básica, com devolutiva e discussão individual de cada encaminhamento ${ }^{(19)}$.

É fundamental a compreensão da operacionalização do programa de atenção integral à saúde da criança no município do Embu para interpretação desses resultados. Cada profissional tem agenda própria, há vinculação com seus pacientes e as consultas são realizadas com registro em prontuários, inclusive as não-agendadas. As fichas da enfermagem contêm informações sobre crescimento e desenvolvimento da criança, também anexadas ao prontuário. Assim, é possível que essa avaliação do desenvolvimento seja realizada no decorrer do acompanhamento da criança e que, devido à presença de outras queixas ou problemas, mesmo em consultas de rotina, o desenvolvimento não seja abordado sempre com a mãe/cuidador com a mesma ênfase; pode ser, ainda, que o médico observe o desenvolvimento da criança, mas não compartilhe dessa avaliação com a mãe/cuidador. Deve-se ressaltar, entretanto, que este compartilhamento é essencial para os familiares ${ }^{(8)}$.

Pittock et al enfatizam a importância da interação entre o processo observacional feito pelo pediatra durante a consulta e o relato dos acompanhantes, que enriquecem a história com dados sobre o comportamento e a evolução da criança no cotidiano, além de poderem confirmar e ajudar o profissional a interpretar os achados de seu exame ${ }^{(10)}$. Nessa perspectiva, as competências dos familiares e dos profissionais de saúde são complementares e estratégicas para o diagnóstico do desenvolvimento das crianças, sendo muito importante que profissionais de saúde procurem explicitar a opinião e as preocupações dos principais cuidadores da criança ${ }^{(22-24)}$. Em um estudo clássico desenvolvido por Glascoe, observou-se que a opinião dos pais quanto ao desenvolvimento dos seus filhos apresentou uma sensibilidade em torno de $80 \%$ no diagnóstico de alterações desse processo ${ }^{(24)}$.

De fato, acredita-se que são necessários programas que visem à atualização dos profissionais e à sensibilização para atuarem de forma mais incisiva em relação à estimulação precoce, orientações antecipatórias, identificação de distúrbios para intervenção oportuna em relação ao desenvolvimento ${ }^{(13,19)}$. A utilização de instrumentos e escalas que pudessem favorecer esse processo tem sido bastante discutida, entretanto, embora o antigo cartão da criança e a atual caderneta apresentem marcos do desenvolvimento, são pouco preenchidos pelos profissionais ${ }^{(25-27)}$. Acredita-se que os instrumentos para avaliar o desenvolvimento possam contribuir para a sistematização dessa avaliação, mas a disponibilidade do profissional para escutar os problemas e questões trazidas pelos familiares é essencial para qualificar a assistência pediátrica e para o efetivo acompanhamento do desenvolvimento da criança. Enfatiza-se o papel do médico da atenção básica como profissional que apresenta contato com a criança em seus primeiros anos de vida, fase na qual é primordial a orientação dos pais para a promoção da saúde, prevenção e detecção de déficits visando à intervenção adequada e oportuna. 
Finalmente, este estudo revelou que os profissionais que prestam assistência à criança nas Unidades Básicas de Saúde do município apresentam algumas falhas no conhecimento e nas práticas relacionadas ao desenvolvimento infantil, reforçando a necessidade de programas de

\section{Referências bibliográficas}

1. Grantham-McGregor S, Cheung YB, Cueto S, Glewwe P, Richter L, Strupp B. Developmental potential in the first 5 years for children in developing countries. Lancet 2007;369:60-70.

2. Silverstein M, Sand N, Glascoe FP, Gupta VB, Tonniges TP, O'Connor KG. Pediatrician practices regarding referral to early intervention services: is an established diagnosis important? Ambul Pediatr 2006;6:105-9.

3. King TM, Glascoe FP. Developmental surveillance of infants and young children in pediatric primary care. Curr Opin Pediatr 2003;15:624-9.

4. Bear LM. Early identification of infants at risk for developmental disabilities. Pediatr Clin North Am 2004;51:685-701.

5. Sand N, Silverstein M, Glascoe F, Gupta VB, Tonniges TP, O'Connor KG. Pediatricians' reported practices regarding developmental screening: do guidelines work? Do they help? Pediatrics 2005;116:174-9.

6. American Academy of Pediatrics; Committee on Children with Disabilities. Developmental surveillance and screening of infants and young children. Pediatrics 2001;108:192-5.

7. Oliveira MC, Napoli S, Liendo S, Belottini C, Contreras M, Aráoz L et al. Consideraciones diagnósticas de los transtornos del espectro autista. Experiencia de un servicio. Med Infant 2008;15:84-94.

8. Halfon N, Regalado M, Sareen H, Inkelas M, Reuland CH, Glascoe FP et al. Assessing development in the pediatric office. Pediatrics 2004;113 (Suppl 6):1926-33.

9. Frankenburg WK. Developmental surveillance and screening of infants and young children. Pediatrics 2002;109:144-5.

10. Pittock ST, Junh YJ, Adegbenro A, Voigt RG. Ease of administration of the cognitive adaptive test/clinical linguistic and auditory milestone scale (CAT/CLAMS) during pediatric well-child visits. Clin Pediatr 2002;41: 397-402.

11. Antonelli CB, Steinhorst F, Ribeiro KC, Chojniak MM, Novaes PE, Arias $V$ et al. O papel do pediatra no diagnóstico precoce do retinoblastoma. Rev Assoc Med Bras 2004;50:400-2.

12. Castilho SD, Costa NA. Diagnóstico precoce do hipotiroidismo congênito: desafio na prática clínica frente a um teste de triagem normal. Rev Cienc Med (Campinas) 2007;16:291-5.

13. Figueiras AC, Puccini RF, Silva EM, Pedromônico MR. Evaluation of practices and knowledge among primary health care professionals in relation to child development surveillance. Cad Saude Publica 2003;19: 1691-9.

14. Zocoli AM, Riecbel FC, Zeigelboim BS, Marques JM. Audição: abordagem do pediatra acerca dessa temática. Rev Bras Otorrinolaringol 2006;72: 617-23. educação permanente, com destaque para a possibilidade de acompanhamento compartilhado entre o médico da atenção básica e os serviços de referência, essenciais no processo de qualificação profissional e na assistência à criança e sua família.
15. Unifesp. Programa de integração docente-assistencial do Embu, 2009 [homepage on the Internet] [cited $2010 \mathrm{Apr}$ 28]. Available from: http://proex. epm.br/novo_site_projetos/programas_projetos.htm

16. Resegue R, Puccini RF, Silva EM. Risk factors associated with developmental abnormalities among high-risk children attendant at a multidisciplinary clinic. Sao Paulo Med J 2008;126:4-10.

17. Seade. Fundação Sistema Estadual de Análise de Dados. Perfil municipal [cited 2010 Apr 28]. Available from: http://www.seade.gov.br/produtos/perfil/ perfil.php

18. Puccini RF, Wechsler R, Silva EM, Resegue R. Fatores de risco em crianças acompanhadas em programas de atenção à saúde da criança. J Pediatr (Rio J) 1997;73:244-51.

19. Figueiras AC. Programa de vigilância do desenvolvimento infantil na atenção primária à saúde - uma experiência no município de Belém [tese de doutorado]. São Paulo: UNIFESP; 2006

20. Lian WB, Ho SK, Yeo CL, Ho LY. General practitioners' knowledge on childhood developmental and behavioural disorders. Singapore Med J 2003;44:397-403.

21. Beggs S, Sewell J, Efron D, Orkin C. Developmental assessment of children: a survey of Australian and New Zealand paediatricians. J Paediatr Child Health 2005;41:444-8.

22. Cardoso RM, Pedromônico MR, Silva EM, Puccini RF. Conhecimento de mães e auxiliares de desenvolvimento infantil referente ao desenvolvimento da linguagem de crianças de zero a vinte e quatro meses. Rev Bras Crescimento Desenvolv Hum 2003;13:85-94.

23. American Academy of Pediatrics; Council on Children With Disabilities Section on Developmental Behavioral Pediatrics; Bright Futures Steering Committee; Medical Home Initiatives for Children With Special Needs Project Advisory Committee. Identifying infants and young children with developmental disorders in the medical home: an algorithm for developmental surveillance and screening. Pediatrics 2006;118:405-20.

24. Glascoe $F$. The value of parents' concerns to detect and address developmental and behavioural problems. J Paediatr Child Health 1999;35:1-8.

25. Figueiras AC, Barros LC, Barlete KC, Faria EC, Fernandes MS, Santos JR. Uso do cartão da criança no município de Belém. Rev Paraense Med 2001;15:39.

26. Alves CR, Lasmar LM, Goulart LM, Alvim CG, Maciel GV, Viana MR et al Quality of data on the child health record and related factors. Cad Saude Publica 2009;25:583-95.

27. Vieira GO, Vieira TO, Costa MC, Santanna Netto PV, Cabral VA. Uso do cartão da criança em Feira de Santana, Bahia. Rev Bras Saude Matern Infant 2005;5:177-84. 